\title{
VARIABILIDAD DE LA FRECUENCIA CARDÍACA COMO INDICADOR DE LA ACTIVIDAD DEL SISTEMA NERVIOSO AUTÓNOMO: IMPLICACIONES EN EL EJERCICIO PATOLOGÍAs.
}

Alvarado Fernández, Víctor'; Camacho Vargas, Shirley'; Monge Rodríguez, Silvia Leticia'; Rodríguez Umaña, David¹ y Fernández Ramírez, Aileen'.

1Departamento de Fisiología de la Escuela de Medicina de la Universidad de Costa Rica, San José, Costa Rica.

Resumen: La variabilidad de la frecuencia cardíaca es uno de los indicadores del funcionamiento del sistema nervioso autónomo y se ha utilizado en la investigación y la clínica como un método no invasivo para determinar su actividad. Su medición se realiza estableciendo parámetros estadísticos de la variación que existe latido a latido en el electrocardiograma, los cuales pueden caracterizarse en dominios temporal y frecuencial. El dominio temporal es la descripción más básica de esta variabilidad y permite obtener el dominio frecuencial. En este último, se calcula un componente de alta frecuencia que se relaciona con la arritmia sinusal respiratoria y el comportamiento de la rama parasimpática; además de un componente de baja frecuencia cuya interpretación ha resultado controversial y podría representar el efecto combinado entre las ramas simpática y parasimpática, moduladas por el barorreflejo. El control neural de la variabilidad de la frecuencia cardíaca involucra los sistemas encefálicos, la médula espinal y los sistemas locales, que son influidos por aferencias de mecano, quimio y barorreceptores. Las aplicaciones del análisis de la variabilidad de la frecuencia cardíaca aún se discuten e involucran una gran variedad de campos como el ejercicio, las enfermedades cardiovasculares y sus factores de riesgo, así como las psicopatologías.

Palabras clave: control de la frecuencia cardíaca, sistema nervioso autónomo, ejercicio, enfermedad cardiovascular. Fuente: MeSH.

Revista electrónica publicada por el Departamento de Farmacología de la Escuela de Medicina de la Universidad de Costa Rica, 2060 San José, Costa Rica. ${ }^{\circledR}$ All rights reserved. Licensed under a Creative Commons Unported License. 


\title{
DETECTION HEART RATE VARIABILITY AS AN INDICATOR OF AUTONOMIC NERVOUS SYSTEM ACTIVATION: IMPLICATIONS IN EXERCISE AND PATHOLOGIES.
}

\begin{abstract}
:
One of the markers of the functioning of the autonomic nervous system is the heart rate variability. It has been used in research and clinical practice as a noninvasive method to determine its activity. Its measurement is made by setting statistical parameters of the variation that exist beat to beat in the electrocardiogram, which can be characterized in time and frequency domains. The time domain is the most basic description of this variability and allows obtaining the frequency domain. In the latter, a high frequency component is measured, which is related with the respiratory sinus arrhythmia and the behavior of the parasympathetic branch. The low frequency component has a controversial interpretation and may represent the combined effect between the sympathetic and parasympathetic branches, modulated by the baroreflex. Neural control of the heart rate variability involves the encephalic system, the spinal cord and local systems, which are influenced by afferences of mechanoreceptors, chemoreceptors and baroreceptors. Applications of the analysis of the heart rate variability are still discussed and involve a wide variety of fields such as exercise, cardiovascular diseases and their risk factors, as well as psychopathologies.
\end{abstract}

Key words: heart rate control, autonomic nervous system, electrocardiography, exercise, cardiovascular disease. Source: MeSH.

\section{INTRODUCCIÓN}

El corazón no late perfectamente regular; el tiempo que transcurre entre latido y latido no es constante, varía debido a la interacción entre el sistema nervioso autónomo (SNA) parasimpático (SP) y simpático (SS), los cuales, son modulados por múltiples factores, desde centros corticales y subcorticales hasta baro y quimiorreceptores periféricos. La variación de la frecuencia cardíaca (VFC) es el cambio de la duración de los intervalos entre latido y latido en el tiempo, e involucra algunos recursos estadísticos que permiten estudiar más a fondo dicha variación $[1,2]$.
Las observaciones iniciales de la VFC, se dieron con la descripción de la arritmia sinusal respiratoria en 1847, en la cual la frecuencia cardíaca aumentaba con la inspiración y disminuía durante la espiración. La primera utilidad clínica se descubrió en 1965, cuando se observó que la disminución de la VFC fetal se asociaba a distress fetal antes de que apareciese algún otro dato clínico sugestivo de éste. De hecho, la monitorización de la VFC fetal aún es parte del protocolo de las unidades de obstetricia mundialmente [3].

En la década de 1980, se publicaron estudios que asociaban la disminución de la VFC con un aumento en la mortalidad, posterior a lo cual se inició una amplia investigación en el campo. El

Revista electrónica publicada por el Departamento de Farmacología de la Escuela de Medicina de la Universidad de Costa Rica, 2060 San José, Costa Rica. ${ }^{\circledR}$ All rights reserved. Licensed under a Creative Commons Unported License. 
primero de ellos, relacionó la disminución de la VFC con una mayor mortalidad posterior a un infarto del miocardio [4]. Este interés llevó a que en 1996 se publicara la primera guía en la cual se establecieron definiciones y metodología para su medición [2], las cuales han sido adoptadas en la mayoría de estudios posteriores. Desde entonces han surgido numerosas investigaciones, muchas con la premisa de que una disminución de la VFC refleja una menor adaptabilidad de la regulación cardiovascular y por lo tanto se asocia con una mayor morbimortalidad [5].

Ante este panorama, el objetivo de esta revisión es presentar los principios fisiológicos que rigen el estudio y la medición de la VFC, así como los factores que la afectan y algunas de las principales utilidades clínicas.

Tabla No. 1. Descomposición espectral de los dominios frecuenciales en el estudio de la VFC $[2,6]$.

\begin{tabular}{ccc}
\hline Frecuencia & $\begin{array}{c}\text { Rangos de } \\
\text { Frecuencia [Hz] }\end{array}$ & $\begin{array}{c}\text { Duración de } \\
\text { periodos }\end{array}$ \\
$\begin{array}{c}\text { Alta [HF] } \\
\text { Baja [LF] }\end{array}$ & $\mathbf{0 , 1 5}-0,4$ & $2,5-6,7 \mathrm{~s}$ \\
$\begin{array}{c}\text { Muy baja } \\
\text { [VLF] }\end{array}$ & $\mathbf{0 , 0 0 3 - 0 , 1 5}$ & $\mathbf{6 , 7 - 2 5 \mathrm { s }}$ \\
$\begin{array}{c}\text { Ultra baja } \\
\text { [ULF] }\end{array}$ & $\leq \mathbf{0 , 0 0 3}$ & $>25 \mathrm{~s}$ \\
\hline
\end{tabular}

\section{MÉTODOS}

Se realizó una búsqueda bibliográfica, utilizando revistas científicas indexadas en Pubmed y Scielo, con los términos de: "Modulación de SNA", "VFC", "medición de la VFC"; a pesar de que se procuró que las fuentes consultadas fuesen de una antigüedad menor a 5 años, se incluyen algunos artículos que se consideran hito en la temática.

Los registros de la VFC se realizaron con el programa Kubios HRV 2.2 y la imagen se creó con Adobe Illustrator CC 2017.

\section{MEDICIÓN DE LA VFC}

La medición de la VFC se realiza a partir de un electrocardiograma (ECG), en el cual se analizan los intervalos $\mathrm{RR}$, es decir, la variación que existe de un latido a otro. En la valoración de la VFC, se utilizan métodos que filtran los latidos prematuros ectópicos y artefactos, detectando y corrigiendo los intervalos RR anormales [6].

Los parámetros estadísticos para la caracterización de la VFC se valoran a partir del dominio temporal o del frecuencial.

\section{Dominio temporal}

Los parámetros de este dominio se expresan en unidades de tiempo (ms), ya que analizan los lapsos entre complejos QRS. Las más utilizadas son: el promedio del intervalo RR (RRi), la desviación estándar de todos los RRi normales (SDNN), la desviación estándar de los promedios de los RRi (SDANN), el promedio de las desviaciones estándar de los RRi en 5 minutos (SNDDi), la raíz cuadrada de la diferencia entre RRi normales adyacentes (rMSSD) y el porcentaje de RRi adyacentes con una diferencia de duración mayor a $50 \mathrm{~ms}$ (pNN50). Estos parámetros sólo ofrecen aspectos muy generales de la variabilidad y no permiten estudiar los ritmos intrínsecos presentes en los latidos cardíacos [6].

\section{Dominio frecuencial}

Estos parámetros permiten cuantificar las fluctuaciones cíclicas del RRi utilizando un análisis de la densidad espectral de potencia, es decir, una descomposición y cuantificación de la VFC en 4 componentes oscilatorios (tabla 1).

Esta técnica de descomposición del registro en bandas de frecuencia es similar a la que se realiza con las señales eléctricas obtenidas con un electroencefalograma. La composición espectral de la VFC no es estática, varía constantemente debido a la interacción de distintos elementos fisiológicos [7].

La intensidad de cada una de estas frecuencias se conoce como potencia o densidad espectral, la cual se expresa en valores absolutos $\left(\mathrm{ms}^{2}\right.$ o s $\left.\mathrm{s}^{2}\right)$ y refleja

Revista electrónica publicada por el Departamento de Farmacología de la Escuela de Medicina de la Universidad de Costa Rica, 2060 San José, Costa Rica. ${ }^{\circledR}$ All rights reserved. Licensed under a Creative Commons Unported License. Contáctenos: rev.med.ucr@gmail.com. Tel: (506) 25-11 4492, Fax: 25-11-4489. 
la varianza de la frecuencia cardíaca en función de las distintas frecuencias, cuantificándose cada componente en un registro continuo de ECG (figura 1) [8].

Existen otros métodos denominados parámetros no lineales con los cuales también se puede analizar la VFC, pero son menos utilizados y su interpretación no es clara [2], por lo que no se abordan en este artículo.

La duración de los ECG que se emplean para la determinación de la VFC se ha estandarizado en 5 minutos o 24 horas, ya que los parámetros pueden variar dependiendo del lapso en el que se miden. En muchos estudios se utilizan registros de otras duraciones (como $2 \mathrm{~min}, 10 \mathrm{~min}, 2 \mathrm{~h}$ ), sin embargo, al analizar los datos es inapropiado comparar registros de distintos rangos temporales. Además, los componentes VLF y ULF no pueden ser determinados en mediciones cortas de 2 o 5 minutos debido a que sus frecuencias oscilan muy lentamente [2].

Usualmente la VFC se refiere a la variabilidad entre los RRi; no obstante, también existe variabilidad de la duración del intervalo QT entre los latidos, lo que ha sido objeto de investigación en los últimos años y se han publicado guías específicas al respecto [9].

La variabilidad del intervalo QT, a una misma frecuencia cardíaca, es un parámetro relacionado con oscilaciones y modificaciones espontáneas en la repolarización ventricular, que se han atribuido a los cambios en la duración del potencial de acción del miocardio y a las características de activación y de velocidad de conducción de los ventrículos [9]. El efecto de las corrientes iónicas sobre la variabilidad latido a latido se ha estudiado en miocitos ventriculares caninos, observándose que las corrientes de sodio, calcio, potasio (rápida y lenta), así como la de potencial transitorio, aumentan la variación del potencial de acción y se relacionan con una mayor probabilidad arritmogénica [10].

\section{Componente de alta frecuencia de la VFC}

Este componente mide la VFC debida a la arritmia sinusal respiratoria. El principal regulador de la arritmia sinusal respiratoria es el SP, por lo que el componente $\mathrm{HF}$ se ha utilizado experimental $\mathrm{y}$ clínicamente como marcador de la actividad del SP $[11,12]$. Esta afirmación se basa en estudios con modelos animales, en los cuales se ha medido directamente la actividad vagal y su relación con la arritmia sinusal respiratoria [12] y en estudios farmacológicos en humanos en los que al realizar bloqueo colinérgico, se disminuye dicha variabilidad $[13,14]$. No obstante, parece ser que la relación del HF con la arritmia sinusal respiratoria se da únicamente en condiciones fisiológicas específicas, las cuales son analizadas extensamente por Grossman et al [14].

Los efectos fisiológicos debidos a la activación o inactivación vagal se observan de manera casi inmediata (latencia aproximada de 0,05 segundos), mientras que los efectos de la activación del SS se registran algunos latidos después (latencia de 5 segundos) [15,16]. Estas diferencias se deben a distintas razones. Por ejemplo, el efecto cronotrópico negativo del SP ocurre por la actividad de los receptores M2 de acetilcolina (ACh) en las células marcapaso del nodo sinusal, donde las subunidades $\beta \gamma$ de la proteína G trimérica asociada favorecen la actividad del canal $\mathrm{IK}_{\mathrm{ACh}}$, con lo cual disminuye la frecuencia de los potenciales de acción [17]. Esta vía de señalización es más rápida, pero el SP también afecta el reloj de calcio y el reloj de membrana de las células marcapaso sinoatriales al disminuir la actividad de la proteína quinasa A (PKA), con lo cual se contrapone a los efectos mediados por el SS que aumenta la PKA [18]. La velocidad de la vía M2$\mathrm{IK}_{\mathrm{ACh}}$ es regulada negativamente por la proteína RGS6 (Regulator of G protein signaling), proteína que se ha señalado como un modulador de la variabilidad intrínseca de la frecuencia cardíaca $[19,20]$. 


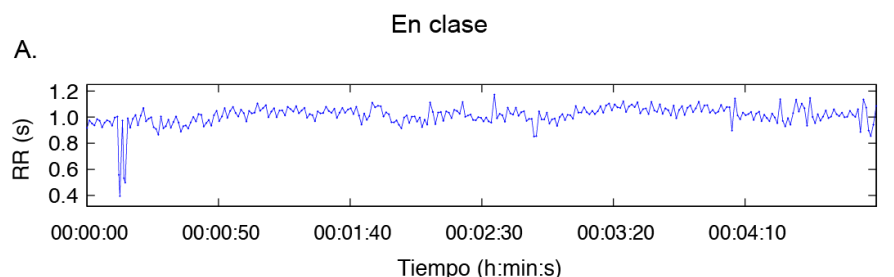

C.
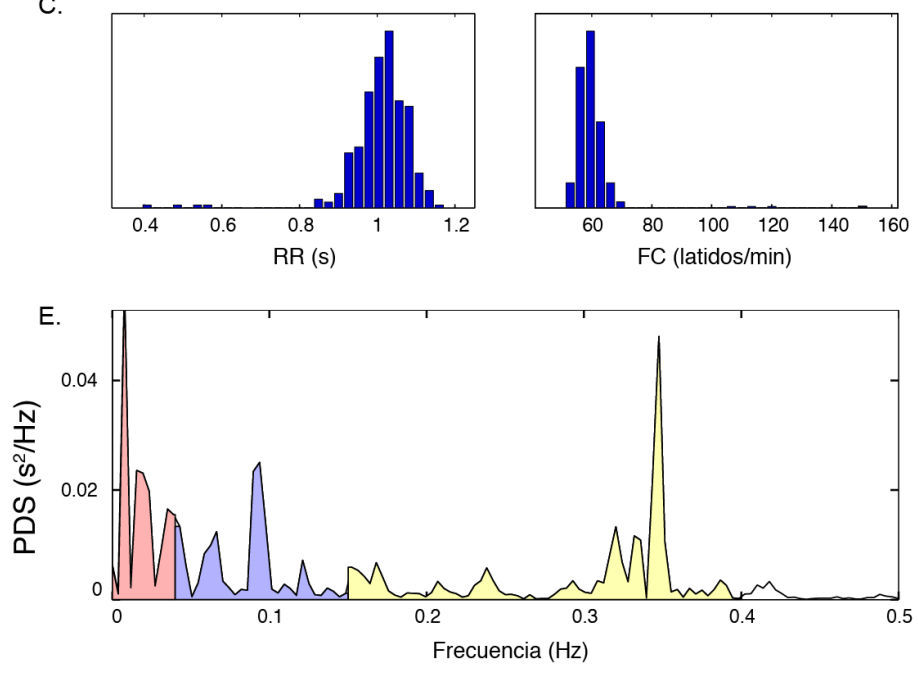

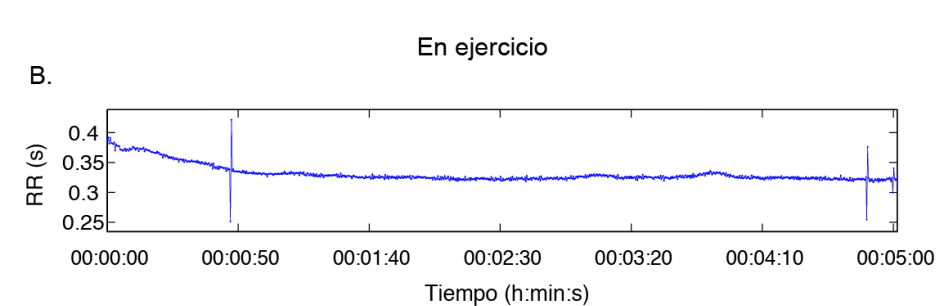

D.
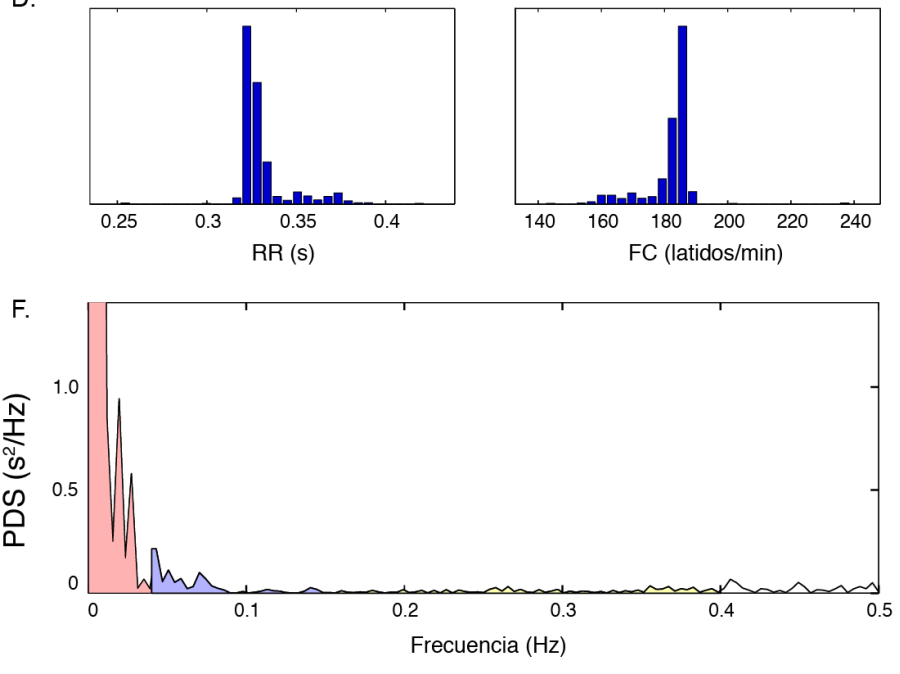

Figura No. 1. Medición e interpretación de VFC. A una estudiante universitaria se le realiza un ECG de 5 minutos cuando se encuentra escuchando una clase y otro cuando se encuentra realizando ejercicio. En el análisis de la VFC se determina la duración de cada RRi durante los 5 minutos y se realiza un tacograma (A. y B.), de los cuales se extraen algunas mediciones del dominio temporal (no se muestra en la imagen) y se grafica la distribución de los RRi y la frecuencia cardiaca (C. y D.). En el ECG y en el tacograma se observa que el RRi varía de latido a latido, de tal forma que a partir de cada RRi se obtiene una frecuencia distinta que se puede expresar en latidos/minutos o en Hertz. De esta manera, la señal inicial (el tacograma) se descompone en VLF, LF y HF. En el análisis de densidad espectral, se cuantifica la distribución en el ECG de cada una de estas frecuencias (E. y F.). Es decir, en el análisis de VFC cuando la estudiante se encontraba escuchando la clase presenta un componente de HF mayor que cuando estaba realizando ejercicio, representando una mayor actividad del SP cuando se encontraba en la clase. ECG: electrocardiograma, RRi: intervalo RR, VFC: variabilidad de la frecuencia cardiaca, VLF: muy baja frecuencia, LF: baja frecuencia, HF: alta frecuencia. SP: sistema parasimpático, PDS: potencia de la densidad espectral. Elaboración propia.

Otro factor implicado en la latencia del SP y relacionado con la VFC es el aclaramiento rápido de la ACh en las sinapsis efectoras, que es mediado por la acetilcolinesterasa, mientras que la norepinefrina (NE) requiere la recaptura de la molécula o su degradación, y este proceso tiene mayor duración [21].
Debido a todo lo anterior, la arritmia sinusal respiratoria y por ende el componente HF pueden ser marcadores de la actividad vagal. La magnitud de la arritmia sinusal respiratoria es influida por otras variables además del SP, dentro de las cuales destacan la frecuencia respiratoria, el volumen corriente, el nivel de actividad física y el estrés. Incluso, algunos estudios han demostrado que el

Revista electrónica publicada por el Departamento de Farmacología de la Escuela de Medicina de la Universidad de Costa Rica, 2060 San José, Costa Rica. ${ }^{\circledR}$ All rights reserved. Licensed under a Creative Commons Unported License. 
bloqueo periférico de receptores adrenérgicos puede afectar el componente HF [22], lo que sugiere un papel del SS, aunque de menor importancia.

Por último, asumir que la frecuencia de disparo del nodo sinusal es un indicador de cómo se encuentra el tono del SNA en otras partes del corazón o el organismo podría no ser correcto, más aún si hay estimulación simultánea del SS y SP [23].

\section{Componente de baja frecuencia de la VFC}

El componente LF ocurre en sincronización con las fluctuaciones fisiológicas de la presión arterial. Inicialmente se postulaba que el componente LF era un marcador directo de la actividad del SS [24], debido a que aumentaba en registros de 24 horas bajo condiciones fisiológicas asociadas al incremento del SS como el estrés y el ejercicio físico [25]. No obstante, luego se reconoció que su origen era más complejo, ya que representa un efecto combinado del SS y el SP [26,27].

Algunos estudios no han mostrado relación entre el componente LF y la tasa de liberación de NE en el corazón [28], ni con la actividad simpática cardíaca $[29,30]$. De hecho, en pacientes con insuficiencia cardíaca, en los que se esperaría una actividad simpática aumentada, se encontró una baja intensidad del componente LF [31].

Por otro lado, el componente LF correlaciona directamente con el barorreflejo, y se ha documentado que la amplitud LF es proporcional a la actividad del barorreflejo [31].

Se ha analizado la relación entre el componente LF y la actividad del SS determinada por medio de la cuantificación de la liberación de NE en el corazón y algunos estudios de medicina nuclear (tomografía con emisión de positrones con 6-(18F) fluorodopamina). Se encontró correlación positiva del componente LF con los estudios de medicina nuclear y la liberación de NE, pero solo en los sujetos que presentaban actividad del barorreflejo normal. Los sujetos con actividad del barorreflejo disminuida mostraron baja intensidad del muscarínico [37]. Costa Rica, 2060 San José, Costa Rica. ${ }^{\circledR}$ All rights reserved. Licensed under a Creative Commons Unported License. Costa Rica, 2060 San José, Costa Rica. ${ }^{\circledR}$ All rights reserved. Licensed under a Creative Commons Unp
(c) Contáctenos: rev.med.ucr@gmail.com. Tel: (506) 25-11 4492, Fax: 25-11-4489.

componente LF y aquellos con actividad normal presentaron la intensidad esperada, independientemente de la actividad simpática que documentaban los demás estudios. Con esto se concluye que el componente LF parece no correlacionar adecuadamente con la actividad simpática, sino con la actividad del barorreflejo [30].

Además, se ha observado que cuando se controlan las respiraciones a una frecuencia de $0,1 \mathrm{~Hz}(6$ respiraciones/minuto), el SP genera oscilaciones de la frecuencia cardíaca que corresponde a los rangos de LF [32]. Es decir, el SP también puede contribuir en el componente LF bajo ciertas circunstancias. Por lo tanto, se cree que el componente LF es una combinación de la actividad simpática, parasimpática y del barorreflejo [1].

\section{Razón LF/HF}

La razón entre el componente LF y HF se ha utilizado para establecer el balance entre el SP y el SS [33], ya que se creía que eran marcadores directos de su nivel de actividad, incluso se había propuesto el rango de 1-2 como cifras normales [34].

Sin embargo, además de la controversia sobre el papel del SS y el SP en los componentes LF y HF, el SS y el SP no actúan siempre de manera recíproca, es decir, que el aumento de la actividad de uno, no siempre se acompaña de la disminución del otro [35]. Por ejemplo, durante el reflejo de buceo aumenta el SS pero se acompaña de una bradicardia profunda [36], la cual se debe a que el aumento de la actividad vagal en el nodo sinusal sobrepasa la actividad adrenérgica generalizada [35].

El aumento o la disminución de la razón $\mathrm{LH} / \mathrm{HF}$ puede deberse a un incremento o decremento desproporcionado de alguna de las dos ramas del SNA. Por lo tanto, la razón puede no aumentar bajo situaciones donde se espera incremento de la actividad simpática, tales como la isquemia cardíaca, el ejercicio o incluso después del bloqueo muscarínico [37]. 


\section{REGULACIÓN NEURAL Y CARACTERÍSTICAS ELECTROFISIOLÓGICAS IMPLICADAS EN LA VFC}

El control neural del corazón involucra a los sistemas encefálicos, reflejos y sistemas locales [7]. Los centros subcorticales implicados en el control de la frecuencia cardíaca son el núcleo motor dorsal del vago y el núcleo ambiguo, vinculados con el SP; y el área ventrolateral rostral del bulbo raquídeo [38], relacionada con la activación del SS. Estas regiones reciben información de mecano, quimio $y$ barorreceptores que modulan su actividad y el balance del SNA [39]. La ínsula, la corteza prefrontal y el hipotálamo representan zonas que procesan estímulos del medio externo e interno y afectan los centros subcorticales de regulación de la frecuencia cardíaca [39-41].

La inervación SP proviene del nervio vago [42] y la mayoría de los axones posganglionares SS del ganglio cervical medio y del ganglio estrellado [43]. El sistema neural cardíaco intrínseco está compuesto por somas y fibras nerviosas interconectadas (plexo ganglionar), de este sistema se extienden nervios epicárdicos que inervan los atrios, el septum interventricular y los ventrículos [44]. La visión clásica del sistema intrínseco cardíaco se relaciona con la activación del SP, este se ha visto como el sitio de sinapsis entre las neuronas pre y postganglionares. Se conoce que puede operar de manera independiente y es capaz de procesar estímulos locales con un amplio perfil neuroquímico $[18,19]$.

Los modelos caninos han mostrado que el sistema intrínseco cardíaco presenta propiedades que podrían ser antiarritmogénicas si es estimulado por el SP $[45,46]$. Se ha observado que el incremento de la actividad de algunos circuitos neurales locales en el corazón favorece la aparición de la fibrilación atrial, y que la estimulación vagal produce un efecto modulador con acciones antiarritmogénicas. Las subpoblaciones del sistema intrínseco cardíaco estimuladas vagalmente conservaron el efecto antiarritmogénico después de que se interrumpieron las aferencias parasimpáticas, por lo que se plantea la existencia de memoria [45].

Revista electrónica publicada por el Departamento de Farmacología de la Escuela de Medicina de la Universidad de Costa Rica, 2060 San José, Costa Rica. ${ }^{\circledR}$ All rights reserved. Licensed under a Creative Commons Unported License.
La estimulación del SS parece influir en el sistema intrínseco cardíaco, ya que al estimular por diferentes periodos la médula espinal (desde T1 hasta T4), se observó un cambio en las características de los potenciales de acción y una mayor eficiencia de las sinapsis de este sistema cuando los estímulos eran prolongados. Este efecto disminuyó al aplicar un bloqueo de los receptores muscarínicos con atropina [46], por lo que, de igual forma podría estar implicada la activación de neuronas del SP o aquellas que liberan ACh como neurotransmisor.

No obstante, la sobreactivación del SS o del SP, favorece la producción de arritmias. Los latidos ectópicos supraventriculares que ocurren ante hiperactividad de alguna rama del SNA han sido predictores del inicio de la fibrilación atrial [47], incluso, se han desarrollado algoritmos de monitorización de la frecuencia cardíaca que predicen dichos eventos [48-51].

Se ha estudiado los potenciales de acción de los miocitos ventriculares izquierdos en modelos caninos utilizando un bloqueo crónico del nodo atrioventricular. Se determinó que la duración del potencial de acción y la variabilidad latido a latido se desacoplaban al disminuir la salida de calcio del retículo sarcoplásmico. Es decir, que la variabilidad latido a latido de este modelo depende mayoritariamente del calcio liberado por el retículo sarcoplásmico [52].

Los factores que influyen en la VFC se pueden clasificar en 5 grupos [7]:

- No modificables, como edad, sexo y etnia.

- Relacionados con el estilo de vida, como ejercicio, tabaco y alcohol.

- Fisiológicos y patológicos, como hormonas sexuales, respiración, presión arterial, asma, daño cerebral y enfermedad cardiovascular.

- Neuropsicológicos como estrés y depresión.

- Ambientales.

\section{FACTORES QUE AFECTAN LA VFC}


Algunos de los más representativos son los efectos dados por el sexo, la etnia, la edad (Tabla 2) y el ejercicio.

\section{Ejercicio y estrés}

Las modificaciones en la VFC debidas al ejercicio físico y al estrés emocional ocurren como consecuencia de un evento agudo o como respuesta adaptativa a un estímulo crónico, como es el caso del entrenamiento físico [64-66].

Existen tres mecanismos implicados en las alteraciones del SNA debidas al control autónomo de la función cardiovascular durante el ejercicio: 1 . el comando central, 2. el mecano o tetanorreflejo (activación de receptores mecanosensibles de los músculos activos, asociados con la inhibición del SP desde que inicia el ejercicio) y, 3. el metaborreflejo o reflejo presor muscular (quimiorreceptores que sensan diversas sustancias liberadas durante la contracción muscular y que se relacionan con el aumento del SS) [66,67]. La información proveniente de estos ergorreceptores musculares viaja por las fibras aferentes del grupo III y IV hacia los núcleos bulbares del centro de control cardiovascular, donde se integran las vías corticales $[67,68]$.

El ejercicio produce una disminución rápida de la VFC (figura 1), principalmente en los primeros 10 a $15 \mathrm{~s}$ de haber iniciado, efecto que se ha relacionado con una inhibición del SP. Conforme avanza el ejercicio y se sobrepasa el umbral de lactato, las modificaciones de la VFC se reducen, debido a una reducción de la contribución de la actividad del SP al control de la frecuencia cardíaca [65,69]. Esta disminución depende de la intensidad y tipo ejercicio que se practica [69].

Cuando se utiliza un ejercicio submáximo como factor estresante para analizar el comportamiento de la VFC es posible distinguir diferentes niveles de la actividad del SNA, dependiendo de la intensidad del ejercicio[70]. Inmediatamente después de finalizar una sesión de intervalos de alta intensidad, el componente HF y la rMSSD disminuyeron más que con una sesión de ejercicio

Revista electrónica publicada por el Departamento de Farmacología de la Escuela de Medicina de la Universidad de Costa Rica, 2060 San José, Costa Rica. ${ }^{\circledR}$ All rights reserved. Licensed under a Creative Commons Unported License. aeróbico, lo que indica una mayor inhibición vagal con el ejercicio intenso; además que la actividad del SP regresa a sus niveles basales en 24 horas, mientras que con el ejercicio moderado existe una recuperación más rápida.

Los autores proponen que el ejercicio de intervalos de alta intensidad produce un mayor estrés en el SNA, elevando el riesgo de eventos cardíacos adversos [71].

En un estudio que mantuvo la frecuencia cardíaca baja y constante, se encontró que los componentes de LF y HF son mayores durante un ejercicio isométrico que durante uno isotónico de extensión de una pierna, por lo que se propone que tanto la actividad vagal, como la simpática son mayores durante el ejercicio isométrico y como consecuencia de esto los efectos del incremento de la actividad simpática serían contrarrestados por una actividad vagal más alta [72]. Los mecanismos fisiológicos responsables de este comportamiento no se conocen.

El entrenamiento físico produce como respuesta adaptativa crónica un incremento de la VFC al analizar los dominios temporales y frecuenciales en diversas poblaciones. En un grupo de adultos sedentarios sometido a un programa de entrenamiento de moderado a vigoroso se encontró un aumento de la pNN50 y de la rMSSD, así como del componente HF [73]. Se han hallado resultados similares en mujeres obesas que realizan actividad física esporádica, siendo las que poseían la mayor capacidad aeróbica aquellas con una mayor VFC [74]. Además, se ha confirmado este efecto del entrenamiento en pacientes con insuficiencia cardíaca, relacionándose con una mejora de su disfunción autónoma [64].

Se ha observado que la capacidad aeróbica influye sobre la rapidez con que se reduce la VFC al inicio del ejercicio, reflejando una mayor inhibición vagal en este periodo en las personas con un mayor consumo máximo de oxígeno ( $\dot{V}_{02} \mathrm{max}$ ), lo que es indicativo de una adaptación positiva generada por 
el entrenamiento [65], que se ha atribuido a una reducción de la actividad vagal y relacionado con una mejoría de la estabilidad eléctrica del miocardio. Por lo tanto, podría ser uno de los mecanismos responsables del efecto beneficioso del ejercicio en la reducción de arritmias cardíacas y muerte súbita [66,73], como se confirmó en un estudio que reportó que existía una relación entre una elevada VFC con una menor mortalidad por cualquier causa y por enfermedades cardiovasculares [75].

Tabla No. 2. Influencia del sexo, la etnia y la edad sobre la VFC.

$\begin{array}{ll}\text { Factor } & \text { Hallazgo } \\ \text { Sexo } & \text { En mujeres con respecto a Ios } \\ {[53,54,} & \text { - Menor intervalo RR. } \\ 55] & \text { - Mayor FC en reposo. } \\ & \text { - Menor SDNN. } \\ & \text { - Menor componente LF. } \\ & \text { - Mayor componente HF. }\end{array}$

Etnia

$[55,56$,

$57,58,59$,

60]

Edad

$[57,60$, 61,62 , 63]

- Mayor VFC en reposo en la etnia negra que en la caucásica.

- Menor componente LF en la etnia negra que en la caucásica.

- Mayor componente HF en la etnia negra que en la caucásica.

- Menor VFC en niños asiáticos que en niños caucásicos en algunos estudios [59], pero en otros no [58].

- Mayor componente LF en niños asiáticos que en niños caucásicos.

- Menor componente HF en niños asiáticos que en niños caucásicos.

- En Latinoamérica son escasas las investigaciones. *

- Existe una disminución gradual significativa de la VFC hasta la 4 ta década. La diferencia entre los dos sexos se pierde después de los 50 años.

- El componente HF disminuye gradualmente hasta la 4ta década.

- El componente LF disminuye a edades avanzadas (después de los 50 años generalmente).

- La razón LF/HF tiende a aumentar con la edad.

* En Costa Rica se llevó a cabo un estudio en donde se intentó definir los parámetros normales de la población; sin embargo, los resultados no fueron confrontados con otras poblaciones. Además se demostró una disminución gradual en la VFC conforme aumenta la edad.

Revista electrónica publicada por el Departamento de Farmacología de la Escuela de Medicina de la Universidad de Costa Rica, 2060 San José, Costa Rica. ${ }^{\circledR}$ All rights reserved. Licensed under a Creative Commons Unported License. 
Todavía no existe claridad en cuanto a la intensidad y tipo de ejercicio más efectivo para maximizar los cambios en la VFC, pero se ha reportado que el elevación de la VFC durante el ejercicio, pero no en reposo [76]. Con el entrenamiento de moderado a vigoroso si se ha observado este efecto en reposo [73] y con el entrenamiento de alta intensidad se ha encontrado un incremento de VFC en reposo y en ejercicio submáximo [77].

En concordancia con estos resultados, se ha reportado una correlación positiva entre el

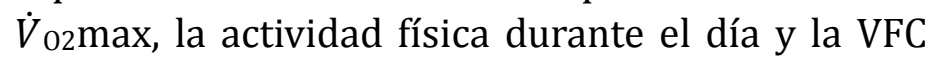
$[65,74]$. En contraposición, se ha concluido que el entrenamiento prolongado de muy alta intensidad, puede generar reducciones de la VFC, por lo tanto, dependiendo de la carga de trabajo al entrenar se establece una curva dosis-respuesta entre la VFC y la intensidad del entrenamiento en forma de campana invertida. Así, se concluyó que el entrenamiento de resistencia de alta intensidad induce un predominio del SS sobre el SP en la modulación autónoma del corazón [78]; pero, no necesariamente implica un efecto negativo del entrenamiento de alta intensidad, aunque sí afecta la utilidad de la VFC para monitorear el efecto del entrenamiento, la capacidad de trabajo y el rendimiento físico de los atletas e implica una monitorización longitudinal e individual para establecer sus valores óptimos y el significado de los mismos en la distribución del entrenamiento [79].

En oposición a los efectos beneficiosos del ejercicio en los índices de VFC, el estrés emocional leve y prolongado en ratas disminuye la VFC en el dominio temporal, mientras que en el dominio estrés crónico aumentaron la potencia del componente LF, reflejando desórdenes de la modulación simpático-vagal, con hiperactivación de SS. Este estado se mantiene en un periodo de recuperación de una semana, reflejando una disfunción autónoma y que podría asociarse con enfermedades cardiovasculares [80]. Por otra entrenamiento de baja intensidad produce una frecuencial se encontró que las ratas expuestas a

parte, se ha reportado que la VFC se reduce en los pasajeros y tripulantes durante los vuelos aéreos, y el impacto de este estrés cardiovascular se reduce conforme aumenta la capacidad aeróbica [81]. Asimismo, en un estudio en Estados Unidos, las personas que presentaban un mayor estrés subjetivo en las actividades de la vida diaria, tenían una VFC menor que los que manejaban mejor las situaciones estresantes. Estos hallazgos respaldan la posibilidad de que la disfunción autónoma puede ser uno de los mecanismos responsables de la asociación entre el estrés diario y la morbimortalidad cardiovascular [82].

Por lo tanto, se evidencia la necesidad de la medición de la VFC como respuesta al ejercicio y al estrés para efectos preventivos, predictivos de enfermedad, terapéuticos o de rendimiento físico en los atletas, sin embargo, está pendiente la estandarización del mejor periodo para realizar estas mediciones, los índices de VFC más apropiados, dependiendo de las características de la persona y el propósito de medición, y la compresión de los mecanismos fisiológicas involucrados en estas respuestas.

\section{APLICACIONES CLÍNICAS DE LA MONITORIZACIÓN DE LA VFC}

La monitorización de la VFC ofrece distintas aplicaciones como un método no invasivo que indica las interacciones entre el SNA y el sistema cardiovascular [6]. Además, las modificaciones de la VFC sugieren una vía común para el aumento de la morbilidad y mortalidad en la patología cardíaca y psiquiátrica [5]. Uno de los primeros estudios en el cual se observó esta asociación, señaló un aumento de la mortalidad de hasta 4 veces conforme disminuía la VFC en pacientes que habían sufrido un infarto agudo al miocardio (IAM). También, determinaron que la tasa de supervivencia era significativamente menor en el grupo de menor variabilidad en un seguimiento de 4 años postinfarto [4].

Asimismo, en una muestra de adultos mayores se observó una asociación entre la disminución de la VFC y el aumento en la mortalidad por cualquier

Revista electrónica publicada por el Departamento de Farmacología de la Escuela de Medicina de la Universidad de Costa Rica, 2060 San José, Costa Rica. ${ }^{\circledR}$ All rights reserved. Licensed under a Creative Commons Unported License.

Contáctenos: rev.med.ucr@gmail.com. Tel: (506) 25-11 4492, Fax: 25-11-4489. 
causa, esto al ajustar por otros factores de riesgo como edad y sexo [83].

Un desequilibrio en el SNA puede ser especialmente perjudicial en pacientes con otros factores de riesgo como diabetes, hipertensión arterial o historia de enfermedad cardiovascular previa, por lo que se ha demostrado que los sujetos diabéticos y con función autonómica alterada tienen el doble de riesgo de mortalidad. Dicha elevación en el riesgo de muerte no se observó en las personas que no presentaban dichas patologías. El aumento en la mortalidad puede atribuirse a que la alteración del SNA incrementa el riesgo de arritmias fatales; sin embargo, esta desregulación podría no ser un factor de riesgo sino una complicación que subyace a la enfermedad cardiovascular y metabólica [84].

El estudio ARIC, demostró que los sujetos con disminución en la VFC tenían un mayor riesgo cardiovascular, de incidencia de enfermedad coronaria y de muerte. Sin embargo, esta condición no pudo ser atribuida a una causa específica ni explicada por otros factores de riesgo, por lo que una baja VFC, en general podría traducirse en un marcador de detrimento en la salud [85]. En el caso de la muerte súbita, varios estudios han demostrado un riesgo aumentado en los sujetos con disminución de la VFC, y especialmente en aquellos post IAM o con insuficiencia cardíaca [88], además se ha asociado a la predisposición a taquiarritmias ventriculares fatales [89].

En este mismo estudio se analizó retrospectivamente la VFC en sujetos que habían sufrido un evento cerebrovascular (ECV) y se determinó una correlación entre una VFC menor y un incremento en el riesgo de sufrir un ECV. Esta relación se dio principalmente en sujetos diabéticos, sugiriendo que es posible que la disfunción autonómica no fue el único factor involucrado. [86]. Otros autores han observado posibles relaciones entre la disminución de la VFC y el ECV, no solo en la mortalidad y funcionalidad a corto y largo plazo, sino también en etiología, Revista electrónica publicada por el Departamento de Farmacología de la Escuela de Medicina de la Universidad de Costa Rica, 2060 San José, Costa Rica. ${ }^{\circledR}$ All rights reserved. Licensed under a Creative Commons Unported License. subtipo, localización e inclusive severidad. Sin embargo, concluyen que son necesarios estudios prospectivos a gran escala para poder

Otro estudio evaluó la relación de la VFC con las escalas de estratificación del riesgo cardiovascular, como las de Hard Coronary Heart Disease, PROCAM, SCORE-CVD y SCORE-CHD. Los resultados demostraron una correlación negativa entre todas las escalas y la VFC, manifestando que a menor VFC, mayor puntaje en cada una de las escalas [90]. En esta misma línea, se ha señalado una asociación de la VFC con la escala de riesgo de Framingham, mostrando diferencias entre los grupos de riesgo medio, alto y muy alto. Por lo tanto, la medición de la VFC ha resultado un método beneficioso y no invasivo, comparado con la determinación de riesgo mediante la escala, la cual requiere análisis de sangre [91].

La hipertensión arterial sistémica (HTA) es otra patología analizada en el estudio ARIC; este asoció bajas VFC en los sujetos que posteriormente desarrollaron HTA, apoyando la noción de que en la génesis de la HTA participa la desregulación del SNA [92]. También, se han desarrollado sistemas automatizados de predicción de eventos cardiovasculares utilizando la VFC para pacientes hipertensos, lo que ha permitido detectar las personas de alto riesgo. Estos sistemas al ser comparados con otros métodos clínicos $y$ ultrasonográficos han demostrado utilidad [93]. Dentro de estas herramientas se presenta la facilidad de poder utilizar estos sistemas en aplicaciones en línea, permitiendo un acercamiento a los clínicos, en la actualidad se realizan estudios de viabilidad [94].

En cuanto a la insuficiencia cardíaca, un estudio demostró mediante un nuevo algoritmo basado en mediciones de corto tiempo (5 minutos), que es posible predecir la incidencia de esta patología, sin embargo, no es posible estratificar la capacidad funcional (estadio NYHA I-IV) ni objetivo de abordaje (clases A-D) [95]. extrapolarlos a la población general [87]. 
Se ha estudiado la relación de la VFC con factores de riesgo cardiovascular a edades tempranas, y se demostró que una disminución de la VFC se asocia a obesidad de predominio abdominal, cifras elevadas de presión arterial e inactividad física; y que los adolescentes con 2 o más factores de riesgo presentaban menor VFC que los que no tenían factores de riesgo [96].

La monitorización de la VFC, además del campo de las patologías cardiovasculares, se ha empleado en otras áreas de la medicina. Por ejemplo, algunos estudios asocian los cambios en la VFC y el balance autonómico con la aparición de migraña, sugiriendo hipofunción parasimpática en esta patología; no obstante, los métodos con los que generalmente se determina la función cardiovascular podrían inducir falsos positivos, por lo que una descripción basada en la VFC sería más representativa del papel de la rama parasimpática en los tipos de migraña que puedan manifestarse, abriendo nuevos campos de exploración [97].

En pacientes con síntomas psicóticos se ha determinado que presentan una baja inhibición en la corteza prefrontal y la amígdala, lo cual se ha asociado a una mayor percepción de amenaza sobre su entorno. Además, poseen una menor actividad vagal cardíaca en reposo y mayor dificultad para contrarrestar los cambios homeostáticos. Los autores sugieren una relación en la aparición de los síntomas psicóticos y brotes paranoides ante factores estresantes, con una baja capacidad de retroalimentación neural. Por lo tanto, se ha señalado que incluso a corto plazo, por medio de una disminución de la frecuencia respiratoria y un aumento de la VFC en reposo, estos pacientes presentan mejoría en los síntomas psiquiátricos, por lo que la monitorización de la VFC podría tener valor pronóstico y terapéutico [98].

Además, en personas que presentan una VFC baja, se observa una mayor tendencia a la desregulación emocional, vulnerabilidad al estrés postraumático y a episodios de angustia relacionados con el aumento de la edad [99].

Revista electrónica publicada por el Departamento de Farmacología de la Escuela de Medicina de la Universidad de Costa Rica, 2060 San José, Costa Rica. ${ }^{\circledR}$ All rights reserved. Licensed under a Creative Commons Unported License.
Del mismo modo, parece estar clara la asociación entre una menor VFC y la depresión o la ansiedad en pacientes con comorbilidad cardíaca. [100]. Los estudios actuales demuestran una relación cada vez más contundente entre la disminución de la VFC en reposo y la aparición de psicopatologías, por lo que es necesaria mayor investigación.

\section{CONCLUSIÓN}

La VFC representa un parámetro que se ha utilizado ampliamente en el estudio del SNA. A pesar que se cuestiona algunos componentes como LF o la relación HF/LF y que se conoce que factores como el sexo, la etnia, la edad y la condición en que se determine el ECG, así como su duración, pueden variar los valores de las mediciones; la VFC es un método no invasivo con el cual se puede obtener un dato personalizado sobre la actividad del SNA, principalmente del SP.

Los mecanismos fisiológicos que subyacen a la VFC comprenden distintos niveles de regulación. La frecuencia cardíaca es el resultado de la integración de múltiples señales que abarcan los elementos celulares en el corazón, los reflejos, los procesos corticales y otros. La investigación creciente de estos temas revela la complejidad de la regulación de esta variable, debido a que integra muchos factores, algunos de los cuales aún se desconocen.

Durante el ejercicio aumenta el tono del SS y disminuye el tono del SP. A corto plazo, el ejercicio disminuye la VFC, efecto que puede tardar 24 horas en regresar a los valores de reposo, dependiendo del tipo y la intensidad del ejercicio. A largo plazo, el ejercicio produce un aumento de la VFC, este efecto beneficioso se ha relacionado con mejor condición aeróbica.

La VFC en el campo clínico ha tenido múltiples asociaciones con psicopatologías, aumento de la mortalidad y con factores de riesgo cardiovascular y metabólicos. Si bien es cierto, no todos los resultados han podido ser replicados y muchas de las utilidades clínicas siguen en estudio, de forma individual podría ser un marcador de riesgo de 
arritmias y correlacionar con la disminución de la adaptabilidad del sistema cardiovascular.

\section{AGRADECIMIENTO}

A la Dra. Catherine Ellis Wegley por la ayuda en la edición de la imagen de este artículo.

\section{BIBLIOGRAFÍA}

1. Shaffer F, Mccraty R, Zerr CL. A healthy heart is not a metronome: an integrative review of the heart's anatomy and heart rate variability. Front Psychol. 2014;5(1040):1-19.

2. Task Force of The European Society of Cardiology and The North American Society of Pacing and Electrophysiology. Guidelines Heart rate variability. Standards of measurement, physiological interpretation, and clinical use. European Heart Journal. 1996.

3. Rajendra U, Joseph PK, Kannathal N, Choo-Min L, Suri JS. Heart Rate Variability. In: Rajendra Acharya U, Suri JS, Spaan JAE, Krishnan SM, editors. Advances in Cardiac Signal Processing. Springer; 2007. p. 121-65.

4. Kleiger RE, Miller JP, Bigger JT, Moss AJ. Decreased Heart Rate Variability and Its Association with Increased Mortality After Acute Myocardial Infarction. Am J Cardiol. 1987;59(4):256-62.

5. Thayer JF, Yamamoto SS, Brosschot JF. The relationship of autonomic imbalance, heart rate variability and cardiovascular disease risk factors. Int J Cardiol. Elsevier Ireland Ltd; 2010;141(2):12231.

6. Draghici AE, Taylor JA. The physiological basis and measurement of heart rate variability in humans. J Physiol Anthropol. 2016;35(22):1-8.

7. Fatisson J, Oswald V. Influence diagram of physiological and environmental factors affecting heart rate variability: an extended literature overview. Hear Int. 2016;11(1):e32-40.

8. Kay SM, Marple SL. Spectrum Analysis-A Modern Perspective. Proc IEEE. 1981;69(11):1380-419.

9. Baumert M, Porta A, Vos MA, Malik M, Couderc J, Laguna $\mathrm{P}$, et al. QT interval variability in body surface ECG : measurement, physiological basis , and clinical QT interval variability in body surface ECG: measurement, physiological basis, and clinical value: position statement and consensus guidance endorsed by the Eur. Europace. 2016;18(6):925-44.

10. Szentandrássy N, Kistamás $K$, Hegyi $B$, Horváth $B$. Contribution of ion currents to beat-to-beat variability of action potential duration in canine ventricular myocytes. Pflugers Arch - Eur J Physiol. 2015;467:1431-43.

11. Fouad FM, Tarazi RC, Ferrario CM, Fighaly S, Alicandri C. Assessment of parasympathetic control of heart rate by a noninvasive method. Am J Physiol. 1984;246(6):H838-42.

12. Katona P, Jih F. Respiratory sinus arrhythmia. J Appl Physiol. 1975;39(5):801-5.

13. Raczkowska M, Eckberg D, Ebert T. Muscarinic cholinergic receptors modulate vagal cardiac responses in man. J Aut Nerv Syst. 1983;3-4:271-8.

14. Grossman P, Taylor EW. Toward understanding respiratory sinus arrhythmia: Relations to cardiac vagal tone, evolution and biobehavioral functions. Biol Psychol. 2007;74:263-85.

15. Badrov MB, Usselman CW, Shoemaker JK. Sympathetic neural recruitment strategies: responses to severe chemoreflex and baroreflex stress. Am J Physiol - Regul Integr Comp Physiol. Bethesda, MD: American Physiological Society; 2015 Jul 15;309(2):R160-8.

16. Eckberg BYDL. Temporal response patterns of the human sinus node to brief carotid baroreceptor stimuli. J Physiol. 1976;258:769-82.

17. He C, Logothetis DE. Identification of a Potassium Channel Site That Interacts with G Protein $\beta \gamma$ Subunits to Mediate Agonist-induced Signaling. J Biol Chem. 1999;274(18):12517-24.

18. Monfredi O, Maltsev VA, Lakatta EG. Modern Concepts Concerning the Origin of the Heartbeat. Physiology. Bethesda, MD: American Physiological Society; 2013 Mar;28(2):74-92.

19. Posokhova E, Ng D, Opel A, Masuho I, Tinker A, Biesecker LG, et al. Essential Role of the m2R-RGS6-I KACh Pathway in Controlling Intrinsic Heart Rate Variability. PLoS One. 2013;8(10):1-10.

20. Wydeven N, Posokhova E, Xia Z, Martemyanov KA, Wickman K. RGS6, but Not RGS4, Is the Dominant Regulator of G Protein Signaling (RGS) Modulator of 
the Parasympathetic Regulation of Mouse Heart Rate. J Biol Chem. 2014;289(4):2440-9.

21. Waller DG, Sampson AP, Renwick AG, Hillier K. Neurotransmission and the peripheral autonomic nervous system. In: Medical pharmacology and therapeutics. 4 ed. Elsevier; 2014.

22. Taylor JA, Myers CW, Halliwill JR, Seidel H, Eckberg DL, Andrew J, et al. Sympathetic restraint of respiratory sinus arrhythmia: implications for vagalcardiac tone assessment in humans. Am J Physiol Hear Circ Physiol. 2001;280(6):2804-14.

23. Inoue H, Zipes DP. Changes in Atrial and Ventricular Refractoriness and in Atrioventricular Nodal Conduction Produced by Combinations of Vagal and Sympathetic Stimulation That Result in a Constant Spontaneous Sinus Cycle Length. Circ Res. 1987;60(6):942-51.

24. Malliani A, Pagani M, Lombardi F, Cerutti S. Cardiovascular Neural Regulation Explored in the Frequency Domain. Circulation. 1991;84:482-92.

25. Brovelli M, Baselli G, Cerutti S, Guzzetti S, Liberati D, Lombardi F, et al. Computerized analysis for an experimental validation of neurophysiological models of heart rate control. Comput Cardiol. 1983;205-8.

26. Akselrod S. Spectral analysis of fluctuations in cardiovascular parameters: a quantitative tool for the investigation of autonomic control. Trends Pharmacol Sci. 1988;9(1):6-9.

27. Appel ML, Berger RD, Saul P, Smith JM, Cohen RJ. Beat to Beat Variability in Cardiovascular Variables: Noise or Music? J Am Coll Cardiol. Elsevier Masson SAS; 1989;14(5):1139-48.

28. Kingwell BA, Thompson JM, Kaye DM, Mcpherson GA, Jennings GL, Esler MD. Heart Rate Spectral Analysis, Cardiac Norepinephrine Spillover, and Muscle Sympathetic Nerve Activity During Human Sympathetic Nervous Activation and Failure. Circulation. 1994;90(1):234-40.

29. Rahman F, Pechnik S, Gross D, Sewell L, Goldstein D. LF Power Reflects Baroreflex Function, Not Cardiac Sympathetic Innervation. Clin Aut Res. 2011;21(3):133-41.

30. Moak JP, Goldstein DS, Eldadah BA, Holmes C, Pechnik S, Sharabi Y. Supine Low Frequency Power of Heart Rate Variability Reflects Baroreflex
Function, Not Cardiac Sympathetic Innervation. Hear Rhythm. 2007;4(12):1523-9.

31. Sleight P, Teresa M, Rovere LA, Mortara A, Pinna G, Maestri R, et al. Physiology and pathophysiology of heart rate and blood pressure variability in humans: is power spectral analysis largely an index of baroreflex gain? Clin Sci. 1995;88:103-1009.

32. Ahmed AK, Harness JB, Mearns AJ, Yorkshire W. Applied Physiology Respiratory Control of Heart Rate. Eur J Appl Physiol. 1982;50:95-104.

33. Pagani M, Lombardi F, Guzzetti S, Sandrone G, Rimoldi O, Malfatto G, et al. Power spectral density of heart rate variability as an index of sympatho-vagal interaction in normal and hypertensive subjects. J Hypertens Suppl. 1984;2(3):S383-5.

34. Sztajzel J. Heart rate variability: a noninvasive electrocardiographic method to measure the autonomic nervous system. Swiss Med Wkly. 2004;134:514-23.

35. Billman GE. The LF / HF ratio does not accurately measure cardiac sympatho-vagal balance. Front Physiol. 2013;4(26):1-5.

36. Fagius J, Sundloft G. The diving response in man: effects on sympathetic activity in muscle and skin nerve fascicles. J Physiol. 1986;377:429-43.

37. Houle MS, Billman GE, Melanie S, Low- GEB. Lowfrequency component of the heart rate variability spectrum : a poor marker of sympathetic activity. Am J Physiol. 1999;276:215-23.

38. Coote JH, Chauhan RA. Autonomic Neuroscience: Basic and Clinical The sympathetic innervation of the heart : Important new insights. Auton Neurosci Basic Clin. Elsevier B.V.; 2016; 199:17-23.

39. Mccraty R, Shaffer F. Heart Rate Variability: New Perspectives on Physiological Mechanisms, Assessment of Self-regulatory Capacity, and Health Risk. Glob Adv Heal Med. 2015;4(1):46-61.

40. Lane RD, Mcrae K, Reiman EM, Chen K, Ahern GL, Thayer JF. Neural correlates of heart rate variability during emotion. Neuroimage. Elsevier B.V.; 2009;44(1):213-22.

41. Nagai M, Hoshide S, Kario K. The insular cortex and cardiovascular system : a new insight into the brainheart axis. J Am Soc Hypertens. 2010;4(4):174-82.

42. Silva LE, Aguiar da Silva CA, Salgado HC, Fazan R. The 
role of sympathetic and vagal cardiac control on complexity of heart rate dynamics. Am J Physiol Hear Circ Physiol. 2017;312(3):H469-H477.

43. Irie T, Yamakawa $\mathrm{K}$, Hamon D, Nakamura K, Vaseghi $\mathrm{M}$, Angeles L, et al. Cardiac Sympathetic Innervation Via the Middle Cervical and Stellate Ganglia and AntiArrhythmic Mechanism of Bilateral Stellectomy. Am J Physiol Hear Circ Physiol. 2017; 312(3): H392-H405.

44. Saburkina I, Gukauskiene L, Rysevaite K, Brack KE, Pauza AG, Pauziene N, et al. Morphological pattern of intrinsic nerve plexus distributed on the rabbit heart and interatrial septum. J Anat. 2014;224(5):583-93.

45. Salavatian S, Beaumont E, Longpré J, Armour JA, Vinet A, Jacquemet $\mathrm{V}$, et al. Vagal stimulation targets select populations of intrinsic cardiac neurons to control neurally induced atrial fibrillation. Am J Physiol Hear Circ Physiol. 2016;311(5):H1311-20.

46. Smith FM, Vermeulen M, Cardinal R. Long-term spinal cord stimulation modifies canine intrinsic cardiac neuronal properties and ganglionic transmission during high-frequency repetitive activation. Physiol Rep. 2016;4(13):1-14.

47. Gallo C, Bocchino PP, Magnano M, Gaido L, Zema D, Battaglia A, et al. Autonomic tone activity before the onset of atrial fibrillation. J Cardiovasc Electrophysiol. 2017;28(3):304-314.

48. Martínez A, Alcaraz R, Rieta JJ. Prediction of paroxysmal atrial fibrillation using recurrence plotbased features of the RR-interval. Physiol Meas. 2011;32(8):1147-62.

49. Chesnokov Y V. Complexity and spectral analysis of the heart rate variability dynamics for distant prediction of paroxysmal atrial fibrillation with artificial intelligence methods. Artif Intell Med. 2008;43(2):151-65.

50. Mohebbi M, Ghassemian H. Predicting termination of paroxysmal atrial fibrillation using empirical mode decomposition of the atrial activity and statistical features of the heart rate variability. Med Biol Eng Comput. 2014;52(5):415-27.

51. Mohebbi M, Ghassemian H, Asl BM. Structures of the Recurrence Plot of Heart Rate Variability Signal as a Tool for Predicting the Onset of Paroxysmal Atrial Fibrillation. J Med Signals Sens. India: Medknow Publications \& Media Pvt Ltd; 2011;1(2):113-21.

52. Antoons G, Johnson DM, Dries E, Santiago DJ,
Ozdemir S, Lenaerts I, et al. Calcium release near L type calcium channels promotes beat-to-beat variability in ventricular myocytes from the chronic AV block dog. J Mol Cell Cardiol. The Authors; 2015;89:326-34.

53. Koenig J, Thayer JF. Sex Differences in Healthy Human Heart Rate Variability: A Meta-Analysis. Neurosci Biobehav Rev. Elsevier Ltd; 2016;64:288310.

54. Dart AM, Du X, Kingwell BA. Gender, sex hormones and autonomic nervous control of the cardiovascular system. Cardiovasc Res. 2002;53:678-87.

55. Allen B, Jennings JR, Gianaros PJ, Thayer JF, Manuck SB. Resting high-frequency heart rate variability is related to resting brain perfusion. Psycophysiology. 2015;52:277-87.

56. Hill LK, Hu D, Koenig J, Sollers III J, Kapuku G, Wang $\mathrm{X}$, et al. Ethnic Differences in Resting Heart Rate Variability: A Systematic Review and Meta-Analysis. Psychosom Med. 2015;77(1):16-25.

57. Liao D, Barnes R, Chambless L, Simpson RJ, Sorlie P, Heiss G. Age, Race and Sex Differences in Autonomic Cardiac Function Measured by Spectral Analysis of Heart Rate Variability - The ARIC Study. Am J Cardiol. 1995;76(12):906-12.

58. Reed K, Warburton D, Whitney C, McKay H. Differences in heart rate variability between Asian and Caucasian children living in the same Canadian community. Appl Physiol Nutr Metab. 2006;31(3):277-82.

59. Eyre ELJ, Fisher JP, Smith EC, Wagenmakers AJM, Matyka KA. Ethnicity and long-term heart rate variability in children. Arch Dis Child. 2013;98(4):292-8.

60. Gutiérrez Sotelo 0. Variabilidad de la frecuencia cardíaca en individuos sanos costarricenses . Revista Costarricense de Cardiología . Scielo; 2000. p. 2-10.

61. Umetani KEN, Singer DH, Craty RMC, Atkinson M. Twenty-Four Hour Time Domain Heart Rate Variability and Heart Rate: Relations to Age and Gender Over Nine Decades. J Am Coll Cardiol. 1998;31(3):593-601.

62. Antelmi I, De Paula R, Shinzato A, Peres C, Mansur A, Grupi C. Influence of Age, Gender, Body Mass Index, and Functional Capacity on Heart Rate Variability in a Cohort of Subjects Without Heart Disease. Am J 
Cardiol. 2004;93(3):381-5.

63. Tsuji H, Vendetti F, Manders E, Evans JC, Larson M, Feldman C, et al. Determinants of Heart Rate Variability. J Am Coll Cardiol. Elsevier Masson SAS; 1996;28(6):1539-46.

64. Hsu C, Hsieh P, Hsiao S, Chien M. Effects of Exercise Training on Autonomic Function in Chronic Heart Failure: Systematic Review. Biomed Res Int. 2015;2015:1-8.

65. D'Agosto T, Peçanha T, Bartels R, Moreira D, Silva L, Nóbrega $\mathrm{A}$, et al. Cardiac Autonomic Responses at Onset of Exercise : Effects of Aerobic Fitness. Int J Sport Med. 2014;35:879-85.

66. Fisher JP. Autonomic control of the heart during exercise in humans: role of skeletal muscle afferents. Exp Physiol. 2014;99(2):300-5.

67. Michelini LC, Leary DSO, Raven PB, Nóbrega ACL. Neural control of circulation and exercise: a translational approach disclosing interactions between central command, arterial baroreflex, and muscle metaboreflex. Am J Physiol Hear Circ Physiol. 2015;309:H381-92.

68. Amann M, Sidhu SK, Weavil JC, Mangum TS, Venturelli M. Autonomic responses to exercise: Group III/IV muscle afferents and fatigue. Auton Neurosci Basic Clin. 2015;188:19-23.

69. Michael S, Jay O, Halaki M, Graham K, Davis GM, Michael S. Submaximal exercise intensity modulates acute post - exercise heart rate variability. Eur J Appl Physiol. Springer Berlin Heidelberg; 2016;116:697706.

70. Pradhapan P, Mika P, Nieminen T, Lehtinen R, Nikus $\mathrm{K}$. Effect of heart rate correction on pre- and postexercise heart rate variability to predict risk of mortality - an experimental study on the FINCAVAS cohort. Front Physiol. 2014;5(208):1-9.

71. Perkins SE, Jelinek HF, Al-aubaidy HA, Jong B De. Immediate and long term effects of endurance and high intensity interval exercise on linear and nonlinear heart rate variability. J Sci Med Sport. Sports Medicine Australia; 2016;1-5.

72. Weippert M, Behrens M, Gonschorek R, Bruhn S. Muscular contraction mode differently affects autonomic control during heart rate matched exercise. Front Physiol. 2015;6(156):1-9.
73. Melanson EL, Freedson PS. The effect of endurance training on resting heart rate variability in sedentary adult males. Eur J Appl Physiol. 2001;85:442-9.

74. Tonello L, Reichert FF, Oliveira-silva I, Rosso S Del. Correlates of Heart Rate Measures with Incidental Physical Activity and Cardiorespiratory Fitness in Overweight Female Workers. Front Physiol. 2016;6(405):1-11.

75. Leeper NJ, Dewey FE, Ashley EA, Sandri M, Tan SY, Hadley D, et al. Prognostic Value of Heart Rate Increase at Onset of Exercise Testing. Circulation. 2007;115:468-75.

76. Martinmäki K, Häkkinen K, Mikkola J. Effect of lowdose endurance training on heart rate variability at rest and during an incremental maximal exercise test. Eur J Appl Physiol. 2008;104:541-8.

77. Leicht AS, Allen GD, Hoey AJ. Influence of Intensive Cycling Training on Heart Rate Variability During Rest and Exercise. Can J Appl Physiol. 2003;28(6):898-910.

78. Iellamo F, Legramante JM, Pigozzi F, Spataro A, Norbiato G, Lucini D, et al. Conversion From Vagal to Sympathetic Predominance With Strenuous Training in High-Performance World Class Athletes. Circulation. 2002;105:2719-25.

79. Plews DJ, Laursen PB, Stanley J, Kilding AE, Buchheit $M$. Training adaptation and heart rate variability in elite endurance athletes: opening the door to effective monitoring. Sport Med. 2013;43(9):773-81.

80. Park SE, Park D, Song K, Seong J-K, Chung S, Youn I. Differential heart rate variability and physiological responses associated with accumulated short- and long-term stress in rodents. Physiol Behav. 2017;115:21-31.

81. Oliveira-silva I, Leicht AS, Moraes MR, Simões HG, Rosso S Del, Córdova C, et al. Heart Rate and Cardiovascular Responses to Commercial Flights: Relationships with Physical Fitness. Front Physiol. 2016;7(648):1-11.

82. Sin NL, Sloan RP, Mckinley PS, Almeida DM. Linking Daily Stress Processes and Laboratory-Based Heart Rate Variability in a National Sample of Midlife and Older Adults. Psychosom Med. 2016;78(5):573-82.

83. Tsuji H, Venditti FJ, Manders ES, Evans JC, Larson MG, Feldman CL, et al. Reduced Heart Rate Variability and Mortalit Risk in an Elderly Cohort 
The Framingham Heart Study. Circulation. 1994;90(2):878-83.

84. Gerritsen J, Dekker J, TenVoorde B, Kostense P, Heine $\mathrm{R}$, Bouter L, et al. Impaired Autonomic Function Is Associated With Increased Mortality, Especially in Subjects With Diabetes, Hypertension or a History of Cardiovascular Disease. Diabetes Care. 2001;24(10):1793-8.

85. Dekker JM, Crow RS, Folsom AR, Hannan PJ, Liao D, Swenne CA, et al. Low Heart Rate Variability in a 2Minute Rhythm Strip Predicts Risk of Coronary Heart Disease and Mortality. Circulation. 2000;102(11):1239-45.

86. Fyfe-johnson AL, Muller CJ, Alonso A, Folsom AR, Gottesman RF, Rosamond WD, et al. Heart rate variability and incident stroke. The Atherosclerosis Risk in Communities Study. Stroke. 2016;47(6):1452-8.

87. Yperzeele L, Hooff R Van, Nagels G, Smedt A De, Keyser J De, Brouns R. Systematic review Heart rate variability and baroreceptor sensitivity in acute stroke: a systematic review. Int J Stroke. 2015;10(6):796-800.

88. Kudaiberdieva G, Görenek B, Timuralp B. Heart rate variability as a predictor of sudden cardiac death. Anadolu Kardiyol Derg. 2007;7(1):68-70.

89. Maheshwari A, Norby FL, Soliman EZ, Adabag S, Whitsel EA, Alonso A, et al. Low Heart Rate Variability in a 2-Minute Electrocardiogram Recording Is Associated with an Increased Risk of Sudden Cardiac Death in the General Population: The Atherosclerosis Risk in Communities Study. PLoS One. 2016;11(8):1-12.

90. Schuster AK, Fischer JE, Thayer JF, Mauss D, Jarczok MN. Decreased heart rate variability correlates to increased cardiovascular risk. Int J Cardiol. Elsevier Ireland Ltd; 2016;203:728-30.

91. Jelinek HF, Imam $\mathrm{H}, \mathrm{Al}$-aubaidy $\mathrm{H}$, Khandoker $\mathrm{AH}$. Association of cardiovascular risk using non-linear heart rate variability measures with the framingham risk score in a rural population. Front Physiol. 2013;4(186):1-9.

92. Schroeder EB, Liao D, Chambless LE, Prineas RJ, Evans GW, Heiss G. Hypertension, Blood Pressure, and Heart Rate Variability. Hypertension. 2003 Dec 11;42(6):1106 LP-1111.
93. Melillo P, Izzo R, Orrico A, Scala P, Attanasio M. Automatic Prediction of Cardiovascular and Cerebrovascular Events Using Heart Rate Variability Analysis. PLoS One. 2015;10(3):1-14.

94. Melillo P, Orrico A, Scala P, Crispino F, Pecchia L. Cloud-Based Smart Health Monitoring System for Automatic Cardiovascular and Fall Risk Assessment in Hypertensive Patients. J Med Syst. 2015;39(10):109.

95. Lucena F, Barros AK, Ohnishi N. The Performance of Short-Term Heart Rate Variability in the Detection of Congestive Heart Failure. Biomed Res Int. 2016;2016:1-11.

96. Ms BQF, Barros MVG, Balagopal B, Ritti-dias RM. Heart Rate Variability and Cardiovascular Risk Factors in Adolescent Boys. J Pediatr. Elsevier Inc; 2014;165(5):945-50.

97. Mamontov O V, Babayan L, Amelin A V, Giniatullin R, Kamshilin AA. Autonomous control of cardiovascular reactivity in patients with episodic and chronic forms of migraine. J Headache Pain. The Journal of Headache and Pain; 2016;17(52):1-8.

98. Clamor A, Koenig J, Thayer JF, Lincoln TM. Behaviour Research and Therapy A randomized-controlled trial of heart rate variability biofeedback for psychotic symptoms. Behav Res Ther. Elsevier Ltd; 2016;87:207-15.

99. Liddell BJ, Kemp AH, Steel Z, Nickerson A, Bryant RA, Tam $N$, et al. Heart rate variability and the relationship between trauma exposure age, and psychopathology in a post-conflict setting. BMC Psychiatry. BMC Psychiatry; 2016;16(133):1-9.

100. Lu W, Tzeng N, Kao Y, Yeh C, Kuo TBJ, Chang C. Correlation between health-related quality of life in the physical domain and heart rate variability in asymptomatic adults. Heal Qual Life Outcomes. Health and Quality of Life Outcomes; 2016;14(149):1-10.

\section{CORRESPONDENCIA}

Alvarado Fernández, Victor

Correo: victor.alvarado@gmail.com 